\title{
APURDUTM
}

Euskal ikerketen aldizkaria | Revue d'études basques |

Revista de estudios vascos | Basque studies review

$3 \mid 1998$

Numéro III

\section{Le renouvellement de la prose basque a travers Buruchkak (1910) de Jean Etchepare}

Jean Casenave

\section{OpenEdition}

\section{Journals}

Édition électronique

URL : https://journals.openedition.org/lapurdum/1710

DOI : 10.4000/lapurdum. 1710

ISSN : 1965-0655

\section{Éditeur}

IKER

Édition imprimée

Date de publication : 1 octobre 1998

Pagination : 221-228

ISBN : 2-84127-152-8

ISSN : $1273-3830$

Référence électronique

Jean Casenave, «Le renouvellement de la prose basque a travers Buruchkak (1910) de Jean

Etchepare », Lapurdum [En ligne], $3 \mid$ 1998, mis en ligne le 01 septembre 2010, consulté le 26 février 2022. URL : http://journals.openedition.org/lapurdum/1710 ; DOI : https://doi.org/10.4000/lapurdum 1710 


\section{J. CASENAVE}

\section{LE RENOUVELLEMENT DE LA PROSE BASQUE A TRAVERS BURUCHKAK (1910) DE JEAN ETCHEPARE}

Jean Etchepare (1877-1935) appartient à la deuxième génération des écrivains basques formés à l'école de la presse. En 1910, il édite un recueil de textes intitulé Buruchkak. Le livre est composé de chroniques déjà publiées dans l'hebdomadaire Eskualduna et d'essais inédits, audacieux par le propos et novateurs au plan littéraire. L'ouvrage est critiqué par la partie la plus conservatrice du public bascophone et l'auteur doit rapidement cesser de le diffuser. Ce rejet pose le problème des relations que l'œuvre en langue basque entretient avec son milieu culturel.

\section{Un contexte culturel favorable}

La période qui couvre la seconde moitié du XIX siècle et le début du $\mathrm{XX}^{\mathrm{e}}$ a été déterminante dans l'histoire de la culture basque moderne. En effet, on y trace des perspectives radicalement nouvelles et on y définit des cadres idéologiques et esthétiques qui sont, pour une grande part, encore les nôtres aujourd'hui. De plus, comme l'ont montré des travaux universitaires récents', c'est durant cette période parfois qualifiée de "Renaissance" que se construit notre littérature moderne par l'introduction de nouveaux genres et notamment le roman, comme par un fort renouvellement des principes esthétiques, dans le domaine de la poésie tout particulièrement.

Sous l'impulsion de quelques fortes personnalités, le monde basque et la langue qui lui est attachée accèdent dans la deuxième moitié du XIX siècle à une véritable existence culturelle. La langue suscite un discours savant au retentissement international. Et c'est, notamment, dans le cadre des célèbres Fêtes basques de la deuxième moitié du XIX ${ }^{\bullet}$ que les acteurs culturels de l'époque s'attachent à définir une identité basque et s'efforcent de lui donner ses attributs distinctifs et ses formes esthétiques propres. C'est donc une véritable imagerie culturelle qui se constitue. La production littéraire écrite apporte une contribution décisive à l'établissement de ce modèle identitaire en se transformant de façon remarquable en l'espace de quelques années.

Au premier rang de ces changements, la mutation linguistique qui s'opère à la fin du $\mathrm{XIX}^{\mathrm{c}}$ siècle en réponse aux exigences de la presse nouvellement créée en langue basque présente un intérêt considérable. Cette mutation suscite dans les provinces du nord du Pays Basque l'apparition du "navarro-labourdin", une forme dialectale élargie comme son nom l'indique. Elle donne naissance, notam- 
ment dans l'hebdomadaire bayonnais l'Eskualduna à une prose fortement renouvelée $^{2}$, novatrice dans sa conception puisqu'adaptée à une nouvelle fonction. Cette évolution linguistique a eu, de surcroît, comme ses pendants guipuscoan ou biscayen, la bonne fortune de connaître une transposition et un couronnement littéraires.

En effet, au milieu de réussites isolées et dans le sillage de Jean Hiriart-Urruty leur maître à écrire, Jean Barbier et Jean Etchepare, les deux écrivains majeurs du domaine navarro-labourdin, ont donné dans cefte forme inter-dialectale nouvellement codifiée des œuvres qui ont aujourd'hui accédé au rang des classiques ( $\mathrm{Su}$ pazter chokoan et Piarres pour le premier nommé, Buruchkak et Beribilez pour le second). La situation culturelle du début du siècle semble propice à la création littéraire. Un vif intérêt pour l'identité, une reconnaissance sociale et scientifique pour la langue, des domaines linguistiques en cours de renouvellement : le développement d'une littérature moderne apparaît comme l'aboutissement tout à fait naturel de ce contexte favorable. Et de fait, grâce au développement de la presse, au retentissement des Fêtes basques et à leurs concours littéraires, la production écrite franchit un palier sur le plan qualitatif comme quantitatif.

\section{Un milieu bascophile divisé}

Cependant, des ombres obscurcissent ce tableau. Ainsi, pour le cercle des érudits et des observateurs étrangers par exemple, la langue basque ne peut pas devenir une langue de culture à part entière. Ils la considèrent comme un objet d'étude certes remarquable mais un code incomplet et à jamais inachevé en quelque sorte, puisqu'il n'a pas donné lieu à une expression littéraire véritablement originale ${ }^{3}$ et que, de plus, il semble en voie d'effacement.

Mais ce point de vue des savants ne représente cependant pas le discours dominant dans le public bascophone. Le groupe qui porte ce discours est organisé autour de l'hebdomadaire l'Eskualduna et s'oppose vigoureusement à ce déclin annoncé même si leur position défensive n'est pas exempte d'un certain pessimisme ${ }^{4}$. Ce groupe possède un journal rédigé pour l'essentiel en basque, un organe de presse autour duquel il a su créer un public de plusieurs milliers de lecteurs réguliers. Cela lui permet de diffuser, à l'intention de la population des campagnes, son modèle identitaire. Celui-ci s'enracine dans le monde rural et se défie de ces phénomènes modernes que sont l'industrialisation et l'urbanisation.

Selon ce modèle, le Basque s’inscrit nécessairement dans la lignée familiale, en respecte les lois, pratique les modes de vie traditionnels et la religion catholique. Liant la pérennité de l'identité à celle ces valeurs, ils conçoivent la langue avant tout comme un "rempart" selon l'expression de J.P. Arbelbide" ou un "isolant" pour reprendre les mots de P. Lhande'. Pour affronter l'avenir et se protéger des influences extérieures jugées préjudiciables à son identité, le monde basque doit selon eux se replier sur lui-même.

C'est sur cet arrière-plan culturel que vient s'inscrire la production littéraire du début $\mathrm{du} X \mathrm{XX}^{\mathrm{e}}$ siècle. Les écrivains de langue basque sont confrontés à un double défi : d'une part, il leur faut faire de leur langue, une langue de culture à part entière en donnant notamment naissance à une expression littéraire de qualité, à la fois moderne et élargie par rapport aux usages conventionnels; d'autre part, ils doivent réaliser une transposition littéraire de ce modèle identitaire que 
le XIX ${ }^{e}$ siècle leur a laissé. Dans le domaine du navarro-labourdin, c'est Jean Etchepare qui, précédant Jean Barbier de quelques années, relève ce défi en publiant en 1910 un recueil de textes intitulé Buruchkak. En fait, ce livre répond implicitement aux deux questions évoquées à propos des débats de la période. Aux lettrés qui considèrent la langue basque comme inapte à se hisser au rang des langues de grande culture, il propose un véritable projet littéraire et apporte la preuve que l'on peut aborder, en langue basque, toutes sortes de sujets même les plus intimistes ou les plus abstraits dans un registre linguistique élevé. Et à l'intention du milieu bascophone partisan de l'identité traditionnelle, Jean Etchepare avance l'image d'une société basque qui cultive ses propres valeurs comme ses représentations esthétiques mais qui s'efforce aussi de les renouveler tout en s'ouvrant aux évolutions du monde extérieur.

\section{L'originalité de Buruchkak.}

Pour composer son livre, Jean Etchepare, reprend quelques-unes des chroniques qu'il a publiées dans l'hebdomadaire bayonnais' entre 1903 et 1909. A ces articles, il joint plusieurs essais inédits dont l'audace du propos et l'orientation résolument littéraire ne passent pas inaperçues. Venons-en maintenant à ce qui fait l'intérêt de cette œuvre. D'abord, il faut noter qu'elle représente, du point de vue chronologique, le premier essai véritablement abouti d'une mise en forme littéraire de la langue codifiée dans l'Eskualduna. Dès les années 1908-1909, l'auteur de Buruchkak disposait d'une production suffisamment riche pour publier un recueil totalement inédit. Or, il retient en définitive un ensemble hétérogène qui mêle des reprises d'articles - retravaillés ${ }^{8}$ dans l'optique de la constitution du recueil - à des textes originaux. En publiant la plupart de ses essais sous forme de chroniques journalistiques dans les deux ans qui précèdent leur parution en volume séparé, le jeune écrivain a voulu procéder à une première socialisation de ses textes et a recherché une forme de validation anticipée. En effet, la stratégie adoptée par l'auteur était destinée, sans doute, à faire accepter les innovations formelles et les points de vue audacieux contenus dans les essais inédits de Buruchkak et notamment le long article consacré à l'enseignement ("Nor eskolaemaile. Zer irakats.") qui proposait, dans une perspective laïque, d'écarter les membres du clergé du domaine éducatif, ainsi que l'essai sur l'amour ("Amodioa") au lyrisme subtil mais trop direct dans ses évocations pour ne pas choquer un milieu sourcilleux sur le plan de la morale sexuelle. Ce choix de mêler anciennes et nouvelles productions est très significatif sur le plan de la communication littéraire. L'effort consenti par l'écrivain pour ajuster son ouvrage à la réalité du champ culturel basque est donc perceptible à travers le processus de composition du recueil et les options stylistiques retenues.

Sans entrer ici dans une étude détaillée du contenu du livre, on peut tout de même indiquer brièvement en quoi Buruchkak renouvelle profondément la production écrite de son temps. On peut retenir quatre innovations importantes. La première concerne le traitement de la thématique basque. Lécrivain travaille la symbolique identitaire par l'intermédiaire de tableaux et de portraits largement tributaires de la veine idéaliste propre à la production littéraire de la deuxième du $\mathrm{XIX}^{\mathrm{e}}$ siècle. On y perçoit également l'influence déterminante de la partie basque de l'œuvre de Pierre Loti. Mais, l'apport de Jean Etchepare ne se limite pas à la transposition esthétique de certains tableaux traditionnels ou à la reprise de ceux 
qui figurent dans Ramuntcho". Il a aussi élargi le cadre thématique habituel en introduisant des sujets jusqu'alors demeurés inédits dans son espace linguistique en abordant des questions d'esthétique, de philosophie morale et même de sociologie. Dans ce premier ouvrage, il a très clairement souhaité élargir son domaine d'écriture en sortant l'espace de quelques essais des cadres jusqu'alors identifiés comme exclusivement basques, en donnant à certains de ses textes une dimension universelle.

La deuxième innovation concerne l'expression de la subjectivité. Celle-ci existe dans la poésie lyrique de la fin du XIX siècle, mais elle demeure très conventionnelle et elle est quasiment absente du domaine de la prose. Jean Etchepare fait d'elle l'un des ressorts de Buruchkak. Rompant avec l'attitude conventionnelle de l'écrivain de langue basque qui s'exprime au nom d'une institution ou se considère comme le porte-voix autorisé de sa communauté, dans Buruchkak, Jean Etchepare se raconte et parle en son nom personnel de façon plus ou moins voilée. Cela passe par le recours au récit autobiographique ou l'adoption d'une position narrative singulière. Quelles que soient les formes de réalisation littéraire, Jean Etchepare accorde dans ses textes une place à l'évocation de l'individu non pas en l'opposant à l'identité collective mais en en faisant un prolongement complémentaire et enrichissant. C'est l'un des incontestables éléments de modernité apportés par l'ouvrage dans le cadre de la littérature basque de l'époque. Ainsi, il transforme la chronique journalistique, son modèle de référence, pour créer des textes plus étendus et plus complexes qui prennent la forme de l'essai ou du récit autobiographique.

Cet élargissement générique s'accompagne d'un intense effort d'adaptation du matériau linguistique disponible dans son domaine dialectal aux besoins d'une expression littéraire ambitieuse et c'est là le troisième élément incontestablement novateur apporté par Jean Etchepare avec Buruchkak. Pour se donner les moyens stylistiques de ses expérimentations, sans perdre de vue les notions de cohérence dialectale et de vraisemblance lexicale, il s'efforce d'élargir les capacités du code linguistique mis au point dans l'Eskualduna. Cependant, on ne peut observer dans ces diverses expérimentations de rupture avec la production immédiatement antérieure puisque Jean Etchepare prolonge l'entreprise d'élargissement et de codification de la langue engagée par la presse, en matière d'orthographe et de création lexicale tout particulièrement. Ainsi, il s'efforce de mêler intelligence de la langue et créativité en expérimentant des formes d'organisation syntaxiques variées et complexes - par exemple ajustées au développement désordonné du monologue intérieur - à partir de modèles déjà connus. Dans Buruchkak, il définit un code littéraire par lequel il contribue de façon décisive à l'établissement de la prose basque moderne.

A côté de la recherche d'un registre littéraire exigeant, ce qui est nouveau dans Buruchkak et ensuite dans l'œuvre critique de l'écrivain, c'est cette réflexion sur le fonctionnement littéraire de la langue, sur son esthétique. Même s'il a considéré jusqu'à la fin de sa vie, le code littéraire établi dans Buruchkak comme une réussite, le travail que Jean Etchepare mène plus tard dans Beribilez ${ }^{10}$ montre bien qu'il s'agissait là pour lui d'un état de langue transitoire. C'est, par exemple, dans un esprit d'expérimentation et non à partir de positions idéologiques ou simplement de logique grammaticale qu'il fait un usage stylistique appuyé du verbe simple ou qu'il élargit les compétences lexicales de son dialecte ou encore tra- 
vaille le langage figuré. Autant d'objectifs qui, retirés de leur contexte, ont valu une réputation de puriste très exagérée à Jean Etchepare.

Enfin, si l'apport stylistique - que certains jugeront donc quelques années plus tard trop élitiste - paraît aujourd'hui fondamental, il n'est cependant pas le seul enjeu formel de Buruchkak. Ce choix d'un registre exigeant le conduit en effet à s'interroger sur la question de la communication littéraire, implicitement dans son ouvrage puis de façon tout à fait ouverte après le retrait du livre. L'écrivain pose les problèmes de l'élaboration, de la diffusion et de la réception de l'œuvre en langue basque en des termes modernes et c'est la quatrième et dernière innovation d'importance apportée par Buruchkak.

S'appuyant sur son expérience de chroniqueur, il élabore un système d'énonciation (formes d'implication du lecteur et marques de la présence de l'auteur) susceptible de capter un public potentiel davantage préparé à la lecture de la presse qu'à celle du texte littéraire. C'est donc également en écrivain qu'il essaie d'évaluer l'état de la situation culturelle et les compétences des lectorats qu'il vise, celui de l'Eskualduna et celui des lettrés. Cela afin d'élaborer un programme de lecture éclectique susceptible de satisfaire tout le monde mais d'où n'est pas exclue une certaine forme de provocation.

Cependant, son ouvrage exigeait en son temps une attitude de lecture nouvelle, plus active qu'à l'ordinaire et plus autonome par rapport aux discours dominants. Or, ce lecteur idéal dont Jean Etchepare sollicite la coopération et même souvent la complicité ne correspond pas au lectorat réel. L'horizon d'attente et les compétences de ce public ne cadrent pas avec le programme littéraire ambitieux proposé par l'auteur de Buruchkak. L'histoire éditoriale du livre indique assez l'importance du fossé qui existait entre le destinataire inscrit dans le texte et le public bascophone courant peu intéressé par les démarches de renouvellement sur le plan des idées comme sur celui des formes littéraires. Depuis les modes d'implication du lecteur dans le discours littéraire jusqu'aux propositions pour améliorer les compétences du lectorat, Jean Etchepare aborde là quelques-uns des thèmes sur lesquels il se retrouvera très isolé tout au long de sa vie, sans doute parce qu'il était en avance sur ses contemporains.

\section{La situation du livre dans la production littéraire de son temps}

Presqu'un siècle après la parution de Buruchkak, la question des difficultés de réception du livre constitue avec la dimension littéraire le point le plus intéressant du dossier critique. Louvrage occupe une position singulière dans le champ culturel basque puisqu'il se situe à la fois dans le cadre et hors du cadre. Il se place dans le cadre car il est produit et publié au cœur même du groupe de l'Eskualduna. Mais, dans le même temps, il se positionne également hors du cadre du fait même de la personnalité de son auteur. De par sa formation de scientifique, sa culture personnelle et sa position de laïc, J. Etchepare s'est forgé une indépendance, un recul critique inhabituel tant au plan des idées que de l'esthétique. C'est cette liberté de penser et d'agir qui l'autorise à introduire dans Buruchkak des thèmes étrangers au monde basque, des thèmes jugés futiles ou poétiques et des sujets tabous.

Cette structure littéraire ouverte et ce mélange des genres, cet intérêt porté aux thèmes les plus élevés comme aux réalités les plus triviales, enfin, cette manière 
de proposer une pluralité de modèles sans en imposer un seul conduisent, toutes proportions gardées, à penser à Montaigne ou à la génération des Lumières. Comme eux, Jean Etchepare a eu le souci de mettre sa pensée au service de la collectivité sans renoncer au plaisir personnel de créer.

Observons maintenant en quels termes cet ouvrage permet de poser la problématique de la création littéraire au début du siècle dans le domaine dialectal du navarro-labourdin. Il s'agit, avant tout, de s'interroger sur la fonction qui dans un tel contexte est assignée à la littérature. En fait, elle paraît conçue en grande partie comme une entreprise d'illustration d'une identité basque qui tend à se replier sur elle-même dans un souci de défense et préservation de valeurs considérées comme, de façon immémoriale, indissolublement liées au patrimoine identitaire. Pour illustrer cette forme de pensée, on peut évoquer l'inclusion systématique des valeurs chrétiennes et rurales à la définition de l'identité basque que pratiquent des auteurs aussi populaires dans le public que J.P. Arbelbide, B. Joannateguy, J. Hiriart-Urruty, J. Moulié ou J. Barbier ${ }^{11}$.

Que, dans ce cadre, la parution de Buruchkak ne manque pas d'apparaître comme une brèche ouverte dans la façade d'un édifice littéraire homogène relève de l'évidence. La véritable question est de savoir si le livre avait quelque chance d'ètre reçu, compris et accepté, s'il pouvait jouer un rôle actif de ferment culturel et littéraire, encourager l'émancipation de la pensée et enfin, s'il pouvait constituer l'amorce d'un courant créateur susceptible de prendre une certaine autonomie par rapport aux références identitaires les plus répandues.

En d'autres termes, cela revient à se demander tout simplement s'il était possible, dans le milieu bascophone du début du siècle, de dire autre chose que ce que ce que proposait le discours dominant? De plus, si l'on considère également le domaine proprement littéraire, un écrivain pouvait-il envisager de considérer la langue basque comme un objet signifiant à part entière, en somme, proposer un usage essentiellement esthétique de ce materiau tout en étant admis par ses contemporains? Ce sont toutes ces questions que posent implicitement les textes de Buruchkak. Le rejet du livre que l'auteur est contraint de cesser de diffuser quelques mois après sa parution indique bien que la réponse à ces questions est négative.

Alors, bien sûr, on doit se demander pourquoi il était irrecevable dans son milieu culturel. Sur le sujet, on possède les explications de P. Lafitte qui indique que l'essai sur l'éducation et notamment la charge contre la présence des prêtres dans l'enseignement avait mécontenté nombre des partisans d'Etchepare. Il ajoute aussi que l'essai sur l'amour avait tout pour choquer malgré les précautions rhétoriques prises par son auteur.

Et pourtant, comment comprendre qu'une communauté qui se déclare menacée de disparition, elle comme sa langue, puisse repousser une œuvre issue de ses rangs, plutôt que de fermer les yeux sur quelques écarts?

\section{Une fonction littéraire en crise}

En fait, ce phénomène de rejet à l'égard d'un texte qui n'a rien d'un pamphlet brutal est le symptôme d'une crise bien plus profonde qui nous conduit à nous interroger à travers Buruchkak sur le rôle que la société bascophone du début du 
siècle assigne à sa production littéraire. Ici, il faut dépasser les conclusions de $\mathrm{P}$. Lafitte très largement liées au contexte passionnel de l'époque et poser le problème en des termes plus techniques à partir de notre conception moderne du texte littéraire.

Comme tous les auteurs marquants, il ouvre des perspectives sur le plan de la pensée, de l'esthétique et de l'expression littéraire. En rejetant cette œuvre qui pourtant le conforte dans sa singularité et le renouvelle, le milieu culturel bascophone local confirme qu'il est bien engagé dans un processus de repli durable. Ce point nous amène donc au deuxième volet de notre interrogation, à savoir les rapports entre l'œuvre littéraire et son lieu d'apparition étudiés à travers l'exemple de Buruchkak. Bien sûr, on peut suivre P. Lafitte lorsqu'il indique que le livre a été censuré pour incompatibilité idéologique et "atteinte aux bonnes mœurs". Mais c'est surtout parce que le milieu bascophone du début du siècle se fait une idée restrictive du rôle de la production écrite que l'ouvrage est rejeté. Les représentants les plus influents de ce milieu conçoivent la littérature comme l'illustration et l'idéalisation du modèle identitaire collectif qu'ils ont contribué à définir. La transposition littéraire se réalise donc sous la forme de récits linéaires très convenus du point de vue esthétique et porteurs de raisonnements simplifiés jusqu'à l'excès dans un souci pédagogique.

Mais en encadrant la création littéraire d'une façon aussi contraignante, en enlevant aux créateurs leur autonomie esthétique et critique, ne se privent-ils pas de possibilités d'évolution? On peut constater que dans le domaine du navarrolabourdin par exemple, au cours des années vingt, c'est la veine créatrice conventionnelle représentée par Jean Barbier qui est mise en valeur. En revanche, on considère la voie proposée par J. Etchepare comme exigeante voire élitiste et de surcroît trop peu orthodoxe. C'est ainsi que tout en maintenant Buruchkak sous l'éteignoir - ni diffusion de la première édition ni réédition d'une version remaniée -, on n'accorde qu'un succès d'estime à Beribilez. Et ce, pendant qu'outre Pyrénées, de jeunes écrivains comme Lizardi ou Lauaxeta s'employaient à faire évoluer le modèle littéraire laissé par la génération de $\mathrm{D}$. Agirre. En définitive, le rejet de Buruchkak représente une forme de rendez-vous manqué en tous points dommageable pour le domaine dialectal du navarro-labourdin. Cependant, Jean Etchepare ne renonce pas. En effet, ce mouvement créateur qui l'a mené des premiers articles à la publication de Buruchkak se poursuit bien après le retrait du livre mais sous une autre forme. Après 1910, l'écrivain tire les leçons de son échec et constate que, dans son milieu culturel, les conditions d'accueil du texte littéraire ne sont pas remplies. C'est pour cela qu'il s'engage dans les institutions culturelles et travaille, tant à la tête de l'Eskualzaleen biltzarra que dans les colonnes de l'Eskualduna, à établir ces conditions de réception.

J. CASENAVE

Docteur en Etudes Basques 


\section{Notes}

1. Jon Kortazar, Teoria y práctica poélica de Esteban Urquiaga, Bilbao, 1986. Ana Toledo, Domingo Agirre: Euskal eleberriaren sorrera. Bilbao, 1989. Karlos Otegi, Lizardi, lectura semiótica de BiotzBegietan, San Sebastián, 1993. Lurdes Otaegi, Lizardiren poetika, Donostia, 1994.

2. Dans chaque domaine dialectal, un processus de codification linguistique accompagne ce mouvement de rénovation de l'écrit.

3. On peut par exemple citer ces propos de Julien Vinson: "Plusieurs écrivains de mérite, français ou espagnols, étaient Basques. Mais il n'existe pas de littérature nationale proprement dite. Les quelques cinq ou six cents volumes en langue basque qui ont pu être répandus dans le pay: sont des traductions du français, de l'espagnol ou du latin ; et le tres petit nombre des ouvrages originaux ont été certainement pensés et écrits par des gens qui avaient reçu une éducation complètement française ou castillane." Les Basques et le Pays basque. Paris, 1882, p.124.

4. Pierre Lhande a exprimé ce point de vue dans son livre Autour d'un foyer basque, Paris, 1908 , p. 147 : “D'autre part, nous rallier à ce progrès qui nous menace, prendre les devants, faire des concessions nous sauvera-t.-1\} davantage?"

5. J.P. Arbelbide, "Aintzin solasak", n. 19, in Igandea edo Jaunaren cruna, Lille, 1895.

6. Pierre Lhande, Autour d'un foyer basque, Paris, 1908, p. 144

7. Jean Etchepare collabore à l'Eskualduna depuis le tout début du siècle (1902-1903).

8. L'étude des divers états du texte permet de suivre l'évolution du texte depuis sa fonction journalistique de type "utilitaire" jusqu'à son accès au statut littéraire

9. Ramuntcho, Paris, 1898 : œuvre inspirée par un long séjour au Pays Basque à Pierre Loti.

10. Jean Etchepare, Beribilez, Bayonne, 1931. Ce récit d'une excursion dans le Pays Basque ne se limite pas à l'évocation des paysages et des villages basques traversés. Jean Etchepare glisse dans ces très belles pages -désormais rangées parmi les classiques de la littérature basque moderne- l'essentiel de ses conceptions philosophiques et esthétiques. Cependant, privilégiant une démarche allusive assez éloignée des procédés littéraires qu'il avait adoptés dans Buruchkak, l'écrivain ne se livre jamais ouvertement dans son second ouvrage.

11. Barbier J, Supazter chokoan, Bayonne, 1924 : "Eta zerk egiten du Eskualduna zinezko Eskualdun? Elizak, Elcheak, Hil -Herriak, Mintzairak. Horietan datza Iskualdun arima guzia."

[Et qu'est-ce qui fait du Basque un véritable Basque ? L'Eglise, la Maison, Le Cimetière, la Langue. En eux se tient toute l'âme basque.] 